\title{
An Electronic Domain Chromatic Dispersion Monitoring Scheme Insensitive to OSNR Using Kurtosis
}

\author{
Kyoung-soo Kim, Jaehoon Lee, and Wonzoo Chung* \\ Division of Computer and Communication Engineering, Korea University, Seoul, 136-701, Korea \\ Sungchul Kim \\ Department of Communication Engineering, Myongji University, Yongin, 448-728, Korea
}

(Received September 29, 2008 : revised November 2, 2008 : accepted November 27, 2008)

\begin{abstract}
In this paper we present an electronic domain solution for chromatic dispersion (CD) monitoring algorithm based on the estimated time domain channel in electronic domain using channel estimation methods. The proposed scheme utilizes kurtosis as a CD measurement, directly computed from the estimated inter-symbol-interference (ISI) channel due to the CD distortion. Hence, the proposed scheme exhibits robust performance under OSNR variation, in contrast to the existing electronic domain approach based on minimum mean squared error (MMSE) fractionally-spaced equalizer taps [1]. The simulation results verify the CD monitoring ability of the proposed scheme.
\end{abstract}

Keywords: Chromatic dispersion monitoring, Electronic domain signal processing, Inter symbol interference, Channel estimation.

OCIS codes : (060.2360) Fiber optics and optical communications: Fiber optics links and subsystems; (060.4510) Fiber optics and optical communications: Optical communications

\section{INTRODUCTION}

During the past decade, optical communication system has realized the rapid growth of data traffic in the network backbone. The capacity increases are contributed by dense wavelength division multiplexing (DWDM) with large channel counts. Optical performance monitoring (OPM) is essential for managing such high capacity optical transmission and switching systems [2]. One of important parameters to estimate is chromatic dispersion (CD), which is a significant factor degrading the performance of optical communication systems [3]. Recently, electronic domain processing approaches have gained considerable research interest to overcome CD and other distortions such as polarization mode dispersion (PMD) [4]. Mature equalization techniques in modern wireless digital communication systems, such as decision feedback equalizers (DFEs) and maximum likelihood sequence estimation (MLSE) equalizers [5, 6] have been successfully applied for mitigation of CD and PMD distortion. In-

*Corresponding author: wchung@korea.ac.kr spired by this electronic domain processing, there has been an attempt to monitor CD in electronic domain [1], unlike other conventional approaches [7, 8]. In [1] authors considered CD monitor scheme based on minimum mean squared error (MMSE) fractionally-spaced (FS) equalizer taps. However, the MMSE FS equalizer taps varies as OSNR changes to deal with noise enhancement [8]. Thus, the proposed monitoring method in [1] reportedly suffers from about $10 \%$ uncertainty when OSNR changes $10 \mathrm{~dB}$ from 30dB. Furthermore, MMSE FS equalizer taps do not directly correspond to the severity of inter-symbol-interface (ISI) channel caused by $\mathrm{CD}$, nor the inverse of channel taps [9]. Hence, the MMSE FS equalizer taps are not the suitable candidate for CD estimation.

In this paper we use kurtosis [10] to estimate the severity of CD directly from the estimate of ISI channel using baud samples. This approach improves the robustness of CD monitor algorithm in the presence of low OSNR and reduces the computational complexity. Since the channel estimation is required by MLSE equalizers and most DFEs for initialization [11,12], this monitoring 
method is a good fit with MLSE equalizers and DFEs for CD and PMD mitigation.

The organization of this paper is as follows. In Section II, we discuss the proposed CD monitoring methods. We describe the relation between CD and ISI and develop a mean to measure ISI for CD monitoring. The performance of the CD monitoring algorithm is confirmed in Section III. Conclusions follow in Section IV.

\section{PROPOSED CD MONITORING METHOD USING ISI}

\section{A. Chromatic dispersion and ISI}

Consider a typical non-return-to-zero on-off-keyed (NRZ-OOK) optical communication model in Figure 1.

The bit sequence $\left\{b_{k}\right\}$ with bit rate $T$ is pulse shaped by a rectangular pulse and transmitted over an optical fiber after laser modulation. We assume a lossless linear fiber with CD distortion. The CD distortion in optical fibers causes generally a non-linear distortion to the transmitted wave forms. However, it is often approximated by a first order distortion model in the optical field domain. The effect of CD can be modeled as a linear filtering process given by

$$
r_{f}(t)=h_{f}(t) \otimes s_{f}(t)
$$

where $s_{f}(t), r_{f}(t)$, and $h_{f}(t)$ are transmitted, received signals, and the fiber impulse response in optical field domain, respectively, and $\otimes$ denotes convolution. In the frequency domain, the transfer function describing fiber $\mathrm{CD}$ is given by the following [5]

$$
H(f)=\exp \left(\frac{j \pi \lambda^{2}}{c} \cdot f^{2} D L\right)
$$

where $L$ denotes the fiber length, $\lambda$ the wavelength, $D$ the dispersion parameter at $\lambda$, and $c$ does speed of light. As the CD parameter increases, the impulse response $h_{f}(t)$ spreads widely and consequently introduces severer inter-symbol-interference (ISI) in the baseband as we will describe in detail shortly. Hence, the severity of ISI can be used as the measure of CD.

The received optical signals suffered from CD distortion are demodulated to electronic signal by a square-law detector. Contrast to the wireless communication sys- tems, this process is nonlinear. Hence, the linear convolution relation in optical field domain is not preserved in the baseband. Nonetheless, The linear component (or the first order approximation) of this nonlinear processing is still written as a convolution and has significant contribution to the overall distortion [13]. Let $h(t)$ denote the impulse response in baseband induced by $h_{f}(t)$ which also includes effect of filtering in optical and electronic domains. Then the received analog signal $r(t)$ in electronic domain can be written as the following [13]

$$
r(t)=\sum_{k=-\infty}^{\infty} b_{k} h(t-k T)+w(t)+\text { non }- \text { linear terms }
$$

where $b_{k}$ denotes the originally transmitted bit sequence, $w(t)$ denotes the noise term, and the non-linear terms appears due to the square-law detector. Assuming optimal sampling timing phase, let $r_{k}$ and $h_{k}$ be the sampled discrete time signal from $r(t)$ and $h(t)$ with respect to the timing period, respectively, i.e.,

$$
\begin{aligned}
& r_{k}=r(k T) \\
& h_{k}=h(k T)
\end{aligned}
$$

Then, we have an end-to-end discrete time domain baseband communication model between the received samples and transmitted bits in the following form

$$
r_{k}=b_{k} \otimes h_{k}+z_{k}
$$

where $z_{k}$ comprises of the sampled noise term and noninear term and $\otimes$ denotes discrete time convolution. Here $h_{k}$ can be viewed as a discrete time impulse response characterizing the optical channel corrupted by CD. In the ideal optical communication scenario, $h_{k}$ satisfies Nyquist criterion [14], i.e.,

$$
h_{k}= \begin{cases}1 & k=0 \\ 0 & k \neq 0\end{cases}
$$

and becomes a pure delay. However, in the presence of $\mathrm{CD}$, the impulse response violates Nyquist criterion and causes inter-symbol-interference (ISI) [14]. The severity of the ISI increases as the CD parameter in (2). To measure ISI it is critical to estimate $\left\{h_{k}\right\}$, since the ISI distortion is given by

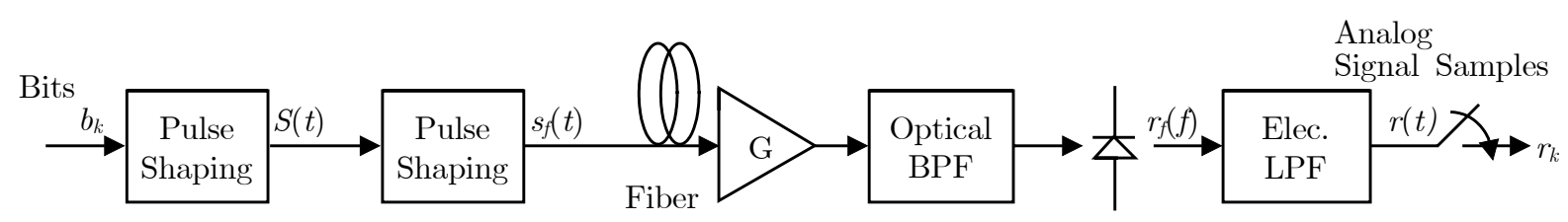

FIG. 1. Fiber communication system using NRZ-OOK 


$$
\begin{aligned}
r_{k} & =b_{k} \otimes h_{k}+z_{k}=\sum_{n=-\infty}^{\infty} b_{n} h_{n-k}+z_{k} \\
& =b_{k} h_{0}+\sum_{n \neq k}^{\infty} b_{n} h_{n-k}+z_{k}
\end{aligned}
$$

where we assumed the 0 delay without loss of generality. The $h_{0}$ term corresponds to the attenuation and non-zero $\left\{h_{k} \mid k \neq 0\right\}$ terms contribute to ISI. As done in wireless digital communication system, we model the $\left\{h_{k}\right\}$ as a finite impulse response (FIR) filter. The vector $h=$ $\left[h_{1}, h_{2}, \cdots, h_{N_{h}}\right]$ is called length $N_{h}$-ISI channel model. Estimating $h=\left[h_{1}, h_{2}, \cdots, h_{N_{h}}\right]$ from the received samples $r_{k}$ is a problem of ISI channel estimation [14,15]. We will apply a classical channel estimation method called adaptive least mean square (LMS) algorithm to estimate $h$ $=\left[h_{1}, h_{2}, \cdots, h_{N_{h}}\right]$. Once the ISI channel is estimated, to measure the severity of ISI channel we propose to use the kurtosis of the estimated channel, which roughly measures the "peakednness" of the channel vector [10].

$$
K(h)=\frac{\sum_{k=1}^{N_{h}}\left|h_{k}\right|^{4}}{\left(\sum_{k=1}^{N_{h}}\left|h_{k}\right|^{4}\right)^{2}}
$$

Apparently, in the absence of ISI, $K(h)$ becomes 1 , and in the worst case when $h_{k}=1$ for all $k, K(h)$ reduces to $1 / N_{h}$, where $N_{h}$ denotes the length of ISI channel.

\section{B. Channel estimation}

We use an adaptive approach to estimate FIR ISI channel $h$ due to CD distortion. Figure 2 illustrate a channel estimation block driven by the decision-directed least mean square (LMS) algorithm [15].

Let $\hat{h}$ denote a vector of length $N_{h}$ to estimate the FIR channel (called channel estimator). To generate error, one can use the originally transmitted signals (for example, training sequences), or decisions $\hat{b_{k}}$ from $r_{k}$ with respect to a decision threshold $\rho$

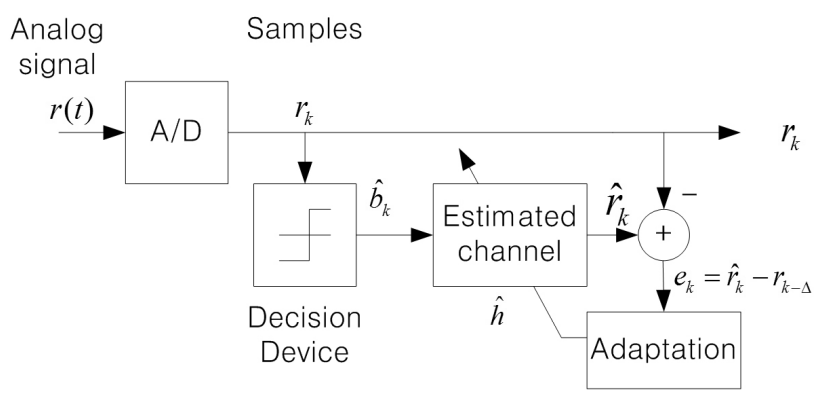

FIG. 2. Decision-directed channel estimation block

$$
\hat{b_{k}}= \begin{cases}1 & r_{k}>\rho \\ 0 & r_{k}<\rho\end{cases}
$$

Based on this information we reconstruct the estimated received signal using $\hat{h}$ :

$$
\hat{r_{k}}=\sum_{k-n=1}^{k-n=N_{h}} b_{n} \hat{h}_{k-n}
$$

The error function is generated by

$$
e_{k}=\hat{r_{k}}-r_{k-\Delta}
$$

where $\Delta$ is the delay. Assuming $\Delta=0$ (in practice $\Delta$ is set to half of the channel estimator length, $N / 2$ ), the mean squared error (MSE) is

$E\left(e_{k}^{2}\right)=E\left(\hat{r}_{k}-r_{k}\right)^{2}=E\left(\sum_{n=1}^{N_{h}} b_{k-n} h_{n}-\sum_{n=1}^{N_{h}} \hat{b}_{k-n} \hat{h}_{n}-z_{k}\right)^{2}$

Further assuming $b_{k}=\hat{b}_{k}$, i.e., no decision error or using training sequence, mean squared error (MSE) can be written as

$$
E\left(e_{k}^{2}\right)=\|h-\hat{h}\|^{2} E\left(b_{k}^{2}\right)+E\left(\text { nonlinar }- \text { terms }^{2}\right)
$$

Hence, the by minimizing MSE we can extract the estimation of the linear component.

$$
h=\hat{h}
$$

The adaptive solution achieving MMSE channel estimation is given by

$$
\hat{h}^{k+1}=\hat{h}^{k}-\mu e_{k} \hat{b_{k}}
$$

where $\mu$ is a step size and $\widehat{h^{k}}$ denotes the estimator vector at $k$-th iteration [14].

Notice that this minimum mean squared error (MMSE) channel estimation is invariant under SNR change in contrast to the fractionally spaced (FS)-MMSE equalizer driven in $[1,9]$, The FS-MMSE is biased to minimize noise enhancement due to inversion of the ISI-channel $[14,15]$, and consequently corresponds to a less severe channel in a low SNR environment. Therefore, severity of ISI measured by a FS-MMSE equalizer is biased in a low SNR as reported in [1].

\section{SIMULATION RESULTS}

In this paper, we simulate optical transmission systems impaired by $\mathrm{CD}$ to verify the $\mathrm{CD}$ monitoring 

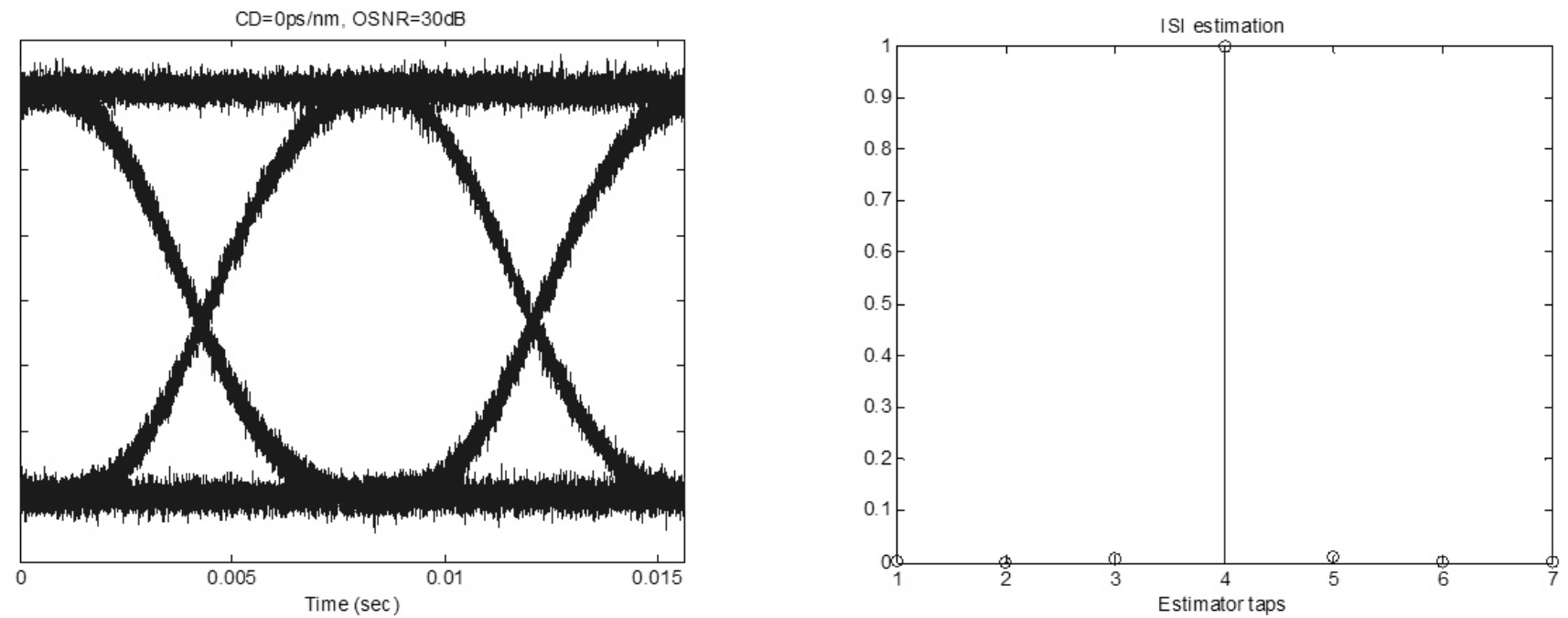

(a) Eye pattern and ISI estimation for $\mathrm{CD}=0 \mathrm{ps} / \mathrm{nm}$ under $30 \mathrm{~dB}$ OSNR
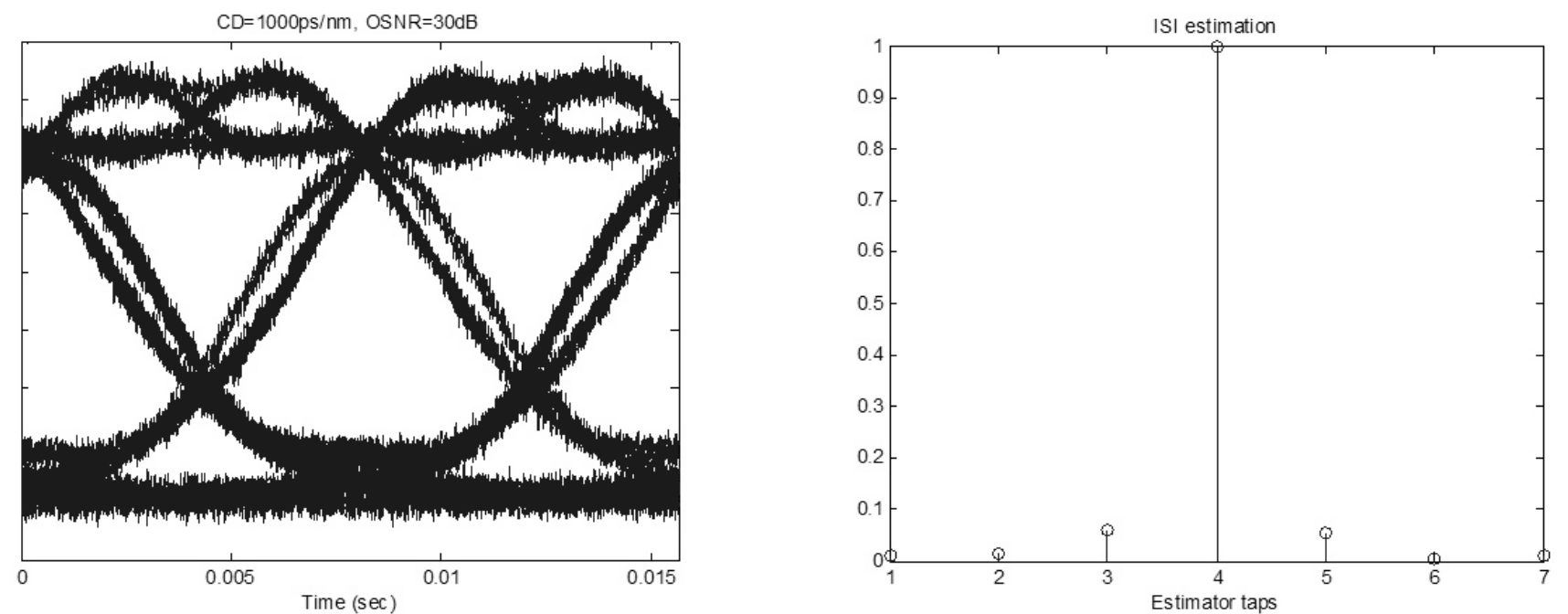

(b) Eye pattern and ISI estimation for $\mathrm{CD}=1000 \mathrm{ps} / \mathrm{nm}$ under 30dB OSNR
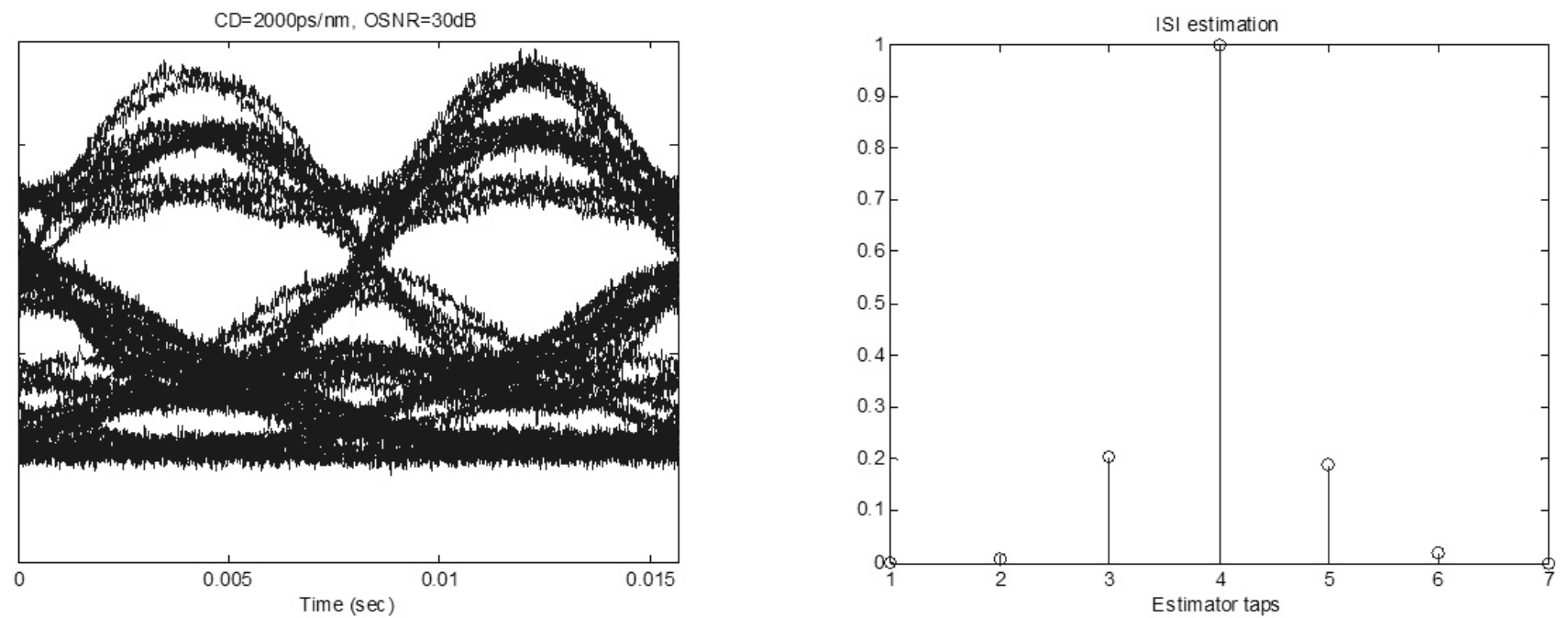

(c) Eye pattern and ISI estimation for $\mathrm{CD}=2000 \mathrm{ps} / \mathrm{nm}$ under $30 \mathrm{~dB}$ OSNR

FIG. 3. Eye pattern and ISI estimation for various CD parameters 


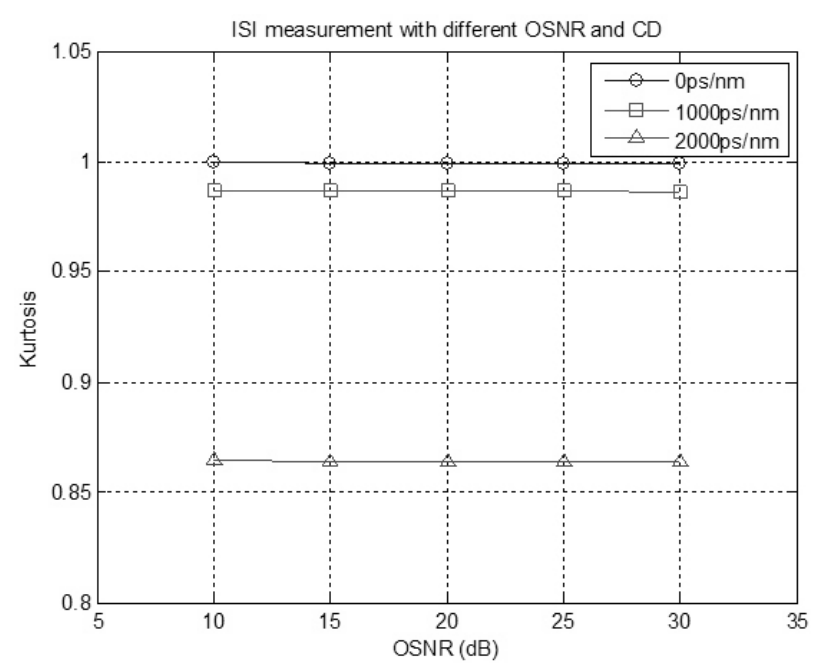

FIG. 4. CD monitoring performance of proposed algorithm under different OSNR

scheme. The transmitter consists of $10 \mathrm{~Gb} / \mathrm{s}$ NRZ-OOK modulation. A CD emulator generates first order $\mathrm{CD}$ distortion. The distorted signal is added with amplified spontaneous emission (ASE) noise. Using square-law detection, electrical signal is obtained from the optical signal. We have used a 5th order Bessel low pass filter with $7 \mathrm{GHz}$ bandwidth after the conversion. The received signal is then fed to the channel estimation block consisted of length 7 channel estimator using least mean square (LMS) algorithm to minimize the MSE. After a few iterations of the LMS algorithm, the estimated channel coefficients are used to calculate the kurtosis. The correlation between the kurtosis of estimated channel and the CD parameter imposed to the optical system is studied by varying the $\mathrm{CD}$ parameter value.

The following Figure 3 (a), (b), (c) show the eyepattern of $0 \mathrm{ps} / \mathrm{nm}, 1000 \mathrm{ps} / \mathrm{nm}$, and $2000 \mathrm{ps} / \mathrm{nm}$ in $30 \mathrm{~dB}$ optical signal-to-noise ratio (OSNR) and ISI estimation using the length 7 channel estimation. The tap coefficients are baud spaced and main cursor is normalized to 1 .

Notice that as CD parameter increases the severity of ISI increases, which indicated by growth of the coefficients values adjacent to the main tap. At $\mathrm{CD}=0$ $\mathrm{ps} / \mathrm{nm}$ the estimator is close to a delta function and vanishingly small ISI is observed. As CD increases to $1000 \mathrm{ps} / \mathrm{nm}$, two adjacent taps increase to about 0.1 and above 0.2 at $\mathrm{CD}=2000 \mathrm{ps} / \mathrm{nm}$.

Figure 4 plots the kurtosis of the ISI estimator computed by (5), as the measurement of $\mathrm{CD}$, for various OSNR and CD. The figure clearly shows that the kurtosis based CD measurement utilizing direct estimation of ISI channel does not vary as OSNR change, in contrast to the $10 \%$ uncertainty reported in [1] when FS-MMSE equalizer coefficients are used to measure CD. Indeed, the CD measurement based on the proposed algorithm exhibits consistent performance under various OSNR.

\section{DISCUSSION AND CONCLUSION}

In this paper, we have proposed a new electronic domain chromatic dispersion monitoring technique using channel estimation. The ISI due to CD is directly estimated from baud samples and measured using kurtosis of the ISI channel. This method has removed the uncertainty of the CD measurement based on the MMSE-FS equalizers under severe OSNR reported in [1]. The successful chromatic dispersion monitoring results have been demonstrated by simulation in a chromatic dispersion range of $1000 \sim 2000 \mathrm{ps} / \mathrm{nm}$.

\section{ACKNOWLEDGMENT}

This work was supported by the Brain Korea 21 project in 2008 .

\section{REFERENCES}

[1] Yi X., Buchali F., Chen W., and Shieh W. "Chromatic dispersion monitoring in electronic dispersion equalizers using tapped delay lines," Opt. Exp., vol. 15, no. 2, pp. 312-315, 2007.

[2] D. C. Kilper, R. Bach, D. J. Blumenthal, D. Einstein, T. Landolsi, L. Ostar, M. Preiss and A. E. Willner, "Optical performance monitoring," J. Lightwave Tech., vol. 22, no. 5, pp. 294-304, 2004.

[3] A. F. Elrefale, R. E. Wagner, D. A. Atlas, and D. G. Daut, "Chromatic dispersion limitations in coherent lightwave transmission systems," J. Lightwave Tech., vol. 6, no. 5, pp. 704-709, 1988.

[4] Savory S. J., "Digital filters for coherent optical receivers", Opt. Exp., vol. 16, no. 2, pp. 804-817, 2008.

[5] Jin Wang and J.M. Kahn, "Performance of electrical equalizers in optically amplified OOK and DPSK systems", IEEE Photon. Technol. Lett., vol. 16, no. 5, pp. 13971399, 2004

[6] Chen W., Buchali F., Yi X., Shieh W., Evans J.S., and Tucker R.S., "Chromatic dispersion and PMD mitigation at $10 \mathrm{~Gb} / \mathrm{s}$ using Viterbi equalization for DPSK and DQPSK modulation formats", Opt. Exp., vol. 15, no. 9, pp. 5271-5276, 2007.

[7] D. C. Kilper, R. Bach, D. J. Blumenthal, D. Einstein, T. Landolsi, L. Ostar, M. Preiss and A. E. Willner, "Optical performance monitoring," J. Lightwave Tech.. vol. 22, no. 1, pp. 294-304, 2004.

[8] A. Liu, G. J. Pendock, and R. S. Tucker, "Chromatic dispersion monitoring using time-multiplexed in-band RF tones," Optical Fiber Communication Conference and Exposition and The National Fiber Optic Engineers Conference, Optical Society of America, OThH6, 2005. 
[9] Ezra Ip and Joseph M. Kahn "Digital Equalization of Chromatic Dispersion and Polarization Mode Dispersion”, J. Lightwave. Tech., vol. 25, no. 8, pp. 2033-2043, 2007.

[10] Darlington, R. B. "Is Kurtosis Really Peakedness?" Amer. Statist. vol. 24, no. 2, pp. 19-22, 1970.

[11] G. D. Forney, "The Viterbi algorithm," Proceedings of the IEEE, vol. 61, no. 3, pp. 268-278, 1973.

[12] J. M. Cioffi, G. P. Dudevoir, M. V. Eyuboglu, and G. D. Forney, "MMSE decision-feedback equalizers and coding-Part I: Equalization results,” IEEE Trans.
Commun., vol. 43, no. 10, pp. 2582-2594, 1995.

[13] W. Rosenkranz, C. Xia, "Electrical equalization for advanced optical communication systems," International Journal of Electronics and Communications, vol. 61, no. 3, pp. 153-157, 2007.

[14] J. Proakis, Digital Communication Systems, (New York, McGraw-Hill Science, 2000).

[15] Treichler, J. R. and Johnson, Jr., C. R. and Larimore, M. G., Theory and Design of Adaptive Filters, (Englewood Cliffs, NJ, Prentice Hall, 2001). 